\title{
THE LAMINAR ORIGIN AND DISTRIBUTION OF THE CROSSED TECTORETICULAR PATHWAYS ${ }^{1}$
}

\author{
VIRGINIA HOLCOMBE ${ }^{2}$ AND WILLIAM C. HALL ${ }^{3}$
}

Department of Anatomy, Duke University Medical Center, Durham, North Carolina 27710

\begin{abstract}
The superior colliculus is the source of a prominent descending pathway which crosses the midline in the mesencephalon and projects to the paramedian pontine reticular formation. The primary goal of the present study was to identify the cells in the superior colliculus of the grey squirrel which give rise to this pathway by using a combination of anterograde and retrograde tracing techniques. Results from the anterograde studies demonstrated the course and terminal distribution of this pathway and suggested that its laminar origin is the intermediate grey layer, stratum griseum intermediale. The retrograde studies were used to confirm the results of the anterograde experiments and to provide a more quantitative estimate of the laminar distribution of the cells which give rise to this pathway. In most cases, over $90 \%$ of the cells retrogradely labeled following injections of horseradish peroxidase along the course of this pathway were located in the intermediate grey lamina. This origin is in contrast to that of the ipsilateral tectoreticular pathway which originates primarily in stratum griseum profundum (Holcombe, V., and W. C. Hall (1981) Neuroscience 6: 255260 ) and suggests that these two grey layers of the deep tectum are functionally distinct.
\end{abstract}

Both anatomy and physiology indicate that the superior colliculus plays an important role in directing gaze toward specific regions in the visual field. For example, stimulation in the intermediate or deep layers elicits saccades, the direction and amplitude of which depend on the site of stimulation. Also, neurons in these layers discharge shortly before saccades to a specific location in the visual field (Schiller and Koerner, 1971; Robinson, 1972; Schiller and Stryker, 1972; Wurtz and Goldberg, 1972; Stryker and Schiller, 1975; Mohler and Wurtz, 1976). In species with a relatively limited range of eye movements, such as the cat, similar relationships are found between collicular activity and movements of the head (Harris, 1980). Correspondingly, anatomical studies have demonstrated major pathways from the superior colliculus to lower brainstem regions known to be involved with the initiation of head and eye movements (Harting et al., 1973; Kawamura et al., 1974; Harting, 1977). The implications of anatomy and physiology receive support from behavioral studies which indicate that

\footnotetext{
${ }^{1}$ This investigation was supported by National Institutes of Health Grant NS-09623 and National Institute of Mental Health Research Scientist Development Award MH-25734. We would like to thank Drs. N. B. Cant and Chia-Sheng Lin for their helpful comments and J. A. Hall for her technical advice. We also thank Ms. Anne Boyd for secretarial assistance.

${ }^{2}$ Current address: Committee on Neurobiology, The University of Chicago, 947 East 58th Street, Chicago, IL 60637.

${ }^{3}$ To whom correspondence should be addressed.
}

ablations of the superior colliculus result in an inability to orient toward stimuli in particular regions of the visual field (Schneider, 1967, 1969; Casagrande and Diamond, 1974; Raczkowski et al., 1976).

Evidence based on stimulating the superior colliculus while recording from neurons in the paramedian pontine reticular formation suggests that the predorsal bundle is the primary pathway through which the superior colliculus influences oculomotor regions of the brainstem. This pathway crosses the midline and descends to terminate monosynaptically on cells in the paramedian reticular formation which, in turn, project to extraocular motor neurons (Grantyn and Grantyn, 1976; Grantyn and Berthoz, 1977; Grantyn et al., 1979). This same region of the reticular formation is apparently also a major source of projections to the cervical spinal cord which may be involved in controlling movements of the head (Kawamura et al., 1974; Peterson et al., 1974; Kuypers and Maisky, 1975; Raybourn and Keller, 1977).

The primary goal of the present study was to identify the cells in the superior colliculus which give rise to this pathway in the grey squirrel (Sciurus carolinensis) by using a combination of anterograde and retrograde tracing techniques. The anterograde results demonstrate the course and terminal distribution of this pathway and suggest its laminar origin. The retrograde studies confirm the results of the anterograde experiments and provide a more precise estimate of the laminar distribution of this pathway's cells of origin. We regard the identification of these cells as a necessary step toward analyzing the 
connections of the superior colliculus which underlie its role in controlling gaze. A preliminary report of these results has been presented in abstract form (Holcombe and Hall, 1978).

\section{Materials and Methods}

In the present experiments, we used adult grey squirrels (Sciurus carolinensis) because of their large, clearly laminated superior colliculi. In all of the experiments, the squirrels were anesthetized with sodium pentobarbital. The lesions and injections were placed stereotaxically. Following the appropriate survival time, each animal was given a lethal dose of barbiturate and perfused through the heart. The brains then were removed and sectioned on a freezing microtome. After selected brain sections were processed, outlines of the sections, which were magnified 15 times, were drawn. The data were plotted onto the outlines with the aid of an X-Y plotter which had been coupled to the stage of a microscope. Cytoarchitectonic borders were confirmed using adjacent unprocessed Nissl-stained sections.

Anterograde axonal degeneration. Electrolytic lesions were produced by passing a DC current (30 to $50 \mu \mathrm{A}$ for 30 to $60 \mathrm{sec}$ ) through a stereotaxically placed stainless steel electrode which was insulated except at its tip. Following postoperative survival times ranging from 2 to 6 days, each animal was perfused with $0.85 \%$ saline followed by $10 \%$ formalin. On the following day, the head was placed in the stereotaxic head holder and the brain was blocked in a coronal plane. The brain then was removed from the skull and stored in $10 \%$ formalin for several days. Following this post-fixation, the brain was transferred to a solution of $10 \%$ formalin and $30 \%$ sucrose. Once adequately infiltrated, the brain was placed on a freezing microtome and sectioned at $30 \mu \mathrm{m}$. Every 10th section through the lesion and the areas of degeneration was stained according to the Fink-Heimer I modification (Fink and Heimer, 1967) of the Nauta reduced silver method. Adjacent sections were stained with cresyl violet.

Anterograde axonal transport. Unilateral injections of equal parts tritiated leucine and lysine $(0.2$ to $0.5 \mu \mathrm{l}$ concentrated in phosphate buffer to $20 \mu \mathrm{Ci} / \mu \mathrm{l}$ ) were made by passing positive current $(0.5 \iota 1.5 \mu \mathrm{A})$ for 3 to $10 \mathrm{~min}$ through a micropipette beveled to a tip opc aing of 15 to $30 \mu \mathrm{m}$. After a $48-\mathrm{hr}$ survival period, each animal was given a lethal dose of sodium pentobarbital and sacrificed by intra-aortic perfusion with $0.85 \%$ saline followed by $10 \%$ buffered formalin. The brain was blocked stereotaxically, removed from the skull, and stored in $10 \%$ buffered formalin for several days. The fixed brain then was transferred to a $10 \%$ formalin solution containing $30 \%$ sucrose. When sufficiently infiltrated with sucrose, the brain was sectioned on a freezing microtome at 30 to 40 $\mu \mathrm{m}$ and sections were collected, mounted on glass slides, dried overnight, and defatted with a graded series of ethanols and xylene (Hendrickson et al., 1972). The slides then were coated with Kodak NTB-3 nuclear track emulsion diluted 1:1 with distilled water and stored in the dark at $4^{\circ} \mathrm{C}$. Following exposure periods ranging from 4 to 8 weeks, the film of emulsion was developed at $13^{\circ} \mathrm{C}$ with Kodak D-19 developer and the sections were stained with cresyl echt violet.
Retrograde axonal transport. Injections of $30 \%$ horseradish peroxidase (HRP) (Boehringer Mannheim) in sterile saline were made either by pressure injection or electrophoresis. Pressure injections were made with a 1$\mu$ l Hamilton syringe, the plunger of which was controlled by a micromanipulator; 0.2 to $0.4 \mu \mathrm{l}$ of HRP solution was deposited over a period of 20 to $30 \mathrm{~min}$. Electrophoretic injections were made by passing a positive current of 0.5 to $1.5 \mu \mathrm{A}$ through a micropipette beveled to a tip opening of 15 to $30 \mu \mathrm{m}$; a constant current was applied for 3 to 6 min. Following a 24-hr survival period, animals were anesthetized deeply and sacrificed by intra-aortic perfusion with a buffered solution of $4 \%$ glutaraldehyde containing $4 \%$ sucrose and $0.5 \%$ polyvinylpyrrolidone. Following sucrose infiltration in a $30 \%$ buffered solution ( 24 to $48 \mathrm{hr}$ at $4^{\circ} \mathrm{C}$ ), the fixed brains were sectioned at $48 \mu \mathrm{m}$ on a freezing microtome and collected in phosphate buffer at $4^{\circ} \mathrm{C}$. Every other section then was reacted with one of three chromagens: diaminobenzidine, benzidine dihydrochloride, or tetramethyl benzidine. The procedures were similar for all three substrates: a 20-min preincubation of the sections in a solution containing the substrate $(100 \mathrm{mg}$ in $200 \mathrm{ml}$ of buffer) was followed by the addition of 2 to $8 \mathrm{ml}$ of $1 \%$ hydrogen peroxide; the sections were agitated gently in this reaction solution for 20 to $25 \mathrm{~min}$ before being rinsed in distilled water at $4^{\circ} \mathrm{C}$, mounted, and counterstained with cresyl echt violet or neutral red.

\section{Results}

Initially, we will describe the overall course and distribution of the contralateral descending fibers which degenerate after a unilateral lesion which involves all laminae of the superior colliculus. Subsequently, the laminar source of this projection system will be estimated based upon a series of amino acid injections, each of which was restricted to only one amina of the superior colliculus. In a final section, a series of cases with HRP injections, which were made at various locations along the course of the contralateral pathway, will be described. This latter series will provide a quantitative estimate of the relative contribution that each lamina makes to the contralateral tectoreticular pathway.

Course and distribution. The case presented in Figure 1 illustrates the major descending pathways which degenerate after a lesion which damages all layers of the superior colliculus. In this case, a caudal approach through the cerebellar cortex was used to make an electrolytic lesion in the right superior colliculus. The lesion involved the caudal half of the superior colliculus and extended from the most superficial lamina (section 57) through the deepest lamina (section 65).

Two major descending pathways are apparent: an ipsilateral and a contralateral tectobulbar pathway. The ipsilateral pathway is not the major topic of this report and will be discussed only in relation to the contralateral projection; the tectopontine subdivision of this ipsilateral pathway has been described in detail in an earlier paper (Holcombe and Hall, 1981b). In the present report, we will restrict our descriptions to the contralateral descending projections to the paramedian reticular formation.

Degenerating axons can be seen descending through the colliculus toward the deepest fibrous lamina, stratum 


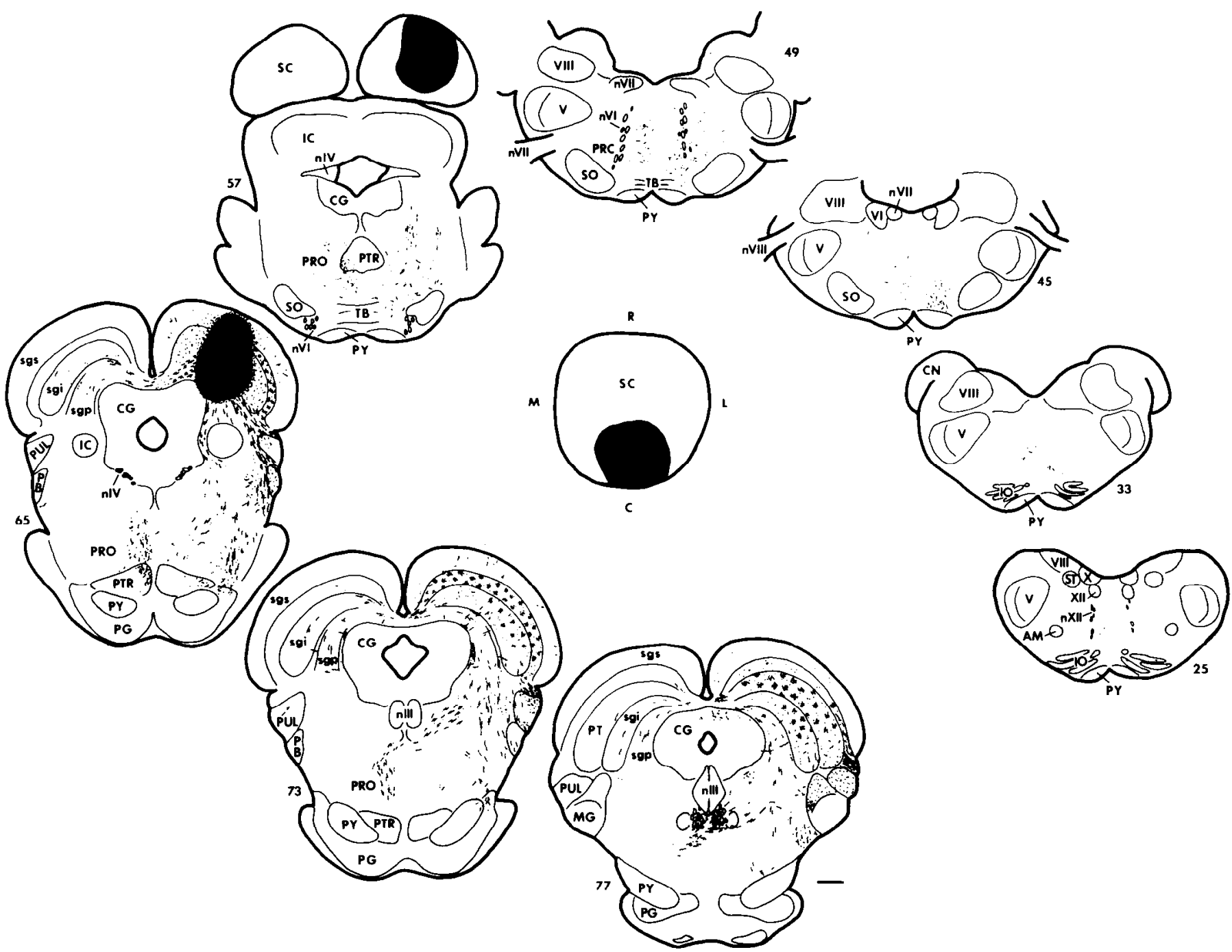

Figure $1 .{ }^{4}$ Drawings of transverse sections through the thalamus and brainstem of a squirrel which received a medium size lesion of the superior colliculus. Degenerating axons are indicated by short lines and terminal degeneration is represented by dots. A surface view of the lesion is shown on the dorsal reconstruction of the colliculus in the center of the figure. Survival time, 4 days; scale, $1 \mathrm{~mm}$.

album profundum, in those sections illustrating more rostral levels of the superior colliculus (sections 65,73 , and 77). Once they reach this fibrous lamina, the axons swing ventrally around the periaqueductal grey area and

\footnotetext{
${ }^{4}$ The abbreviations used on the figures are: AM, nucleus ambiguus; $\mathrm{C}$, caudal; CG, central grey; $\mathrm{CN}$, cochlear nucleus; contra, contralateral; $\mathrm{CP}$, cerebral peduncle; DGL, dorsal lateral geniculate nucleus; IC, inferior colliculus; IO, inferior olive; ipsi, ipsilateral; L, lateral; $M$, medial; MG, medial geniculate nucleus; n.III, oculomotor nerve; n.IV, trochlear nerve; $n$.VI, abducens nerve; n.VII, facial nerve; n.VIII, auditory-vestibular nerve; n.XII, hypoglossal nerve; OT, optic tract; $\mathrm{PB}$, parabigeminal nucleus; $\mathrm{PG}$, pontine grey; $\mathrm{PN}$, pontine nuclei; PRC, pontine reticular nucleus, caudal part; PRO, pontine reticular nucleus, oral part; PT, pretectum; PTR, pontine tegmental reticular nucleus; PUL, pulvinar; PY, pyramidal tract; $R$, rostral; RN, red nucleus; $S C$, superior colliculus; sgs or s, stratum griseum superficiale; sgi or i, stratum griseum intermediale; sgp or $\mathbf{p}$, stratum griseum profundum; SO or SOC, superior olivary complex; ST, solitary nucleus; TB, trapezoid body; t.V, spinal tract of the trigeminal nucleus; III, oculomotor nucleus; V, trigeminal nucleus; VI, abducens nucleus; VII, facial nucleus; VIII, vestibular nuclei; X, motor nucleus of the vagus; XII, hypoglossal nucleus.
}

turn rostrally. They then proceed as far rostral as the oculomotor nuclei before crossing the midline in the dorsal tegmental decussation. As they cross, the fibers penetrate at right angles the descending oculomotor nerve (sections 73 and 77).

After decussating, the degenerating axons change direction abruptly and course ventrally within the contralateral midbrain tegmentum (section 73). This prominent bundle of degenerating fibers projects toward the pontine tegmental reticular nucleus and heavy terminal degeneration is present in the most medial part of this nucleus (section 65).

Caudal to this nucleus, the fibers travel caudally within fascicles and maintain a position near the midline throughout the brainstem. As the pathway continues, individual degenerating axons peel away from these fascicles and terminate within the adjacent reticular formation. Most of the degeneration is confined to the medial third of the tegmentum and is present within both oral and caudal subdivisions of the pontine reticular nucleus (sections 65, 57, and 49).

The number of degenerating axons decreases dramat- 
ically near the pontomedullary junction. Only very sparse terminal degeneration is present within the paramedian medullary tegmentum and, in the case illustrated here, only one or two degenerating axons could be traced as far caudally as the spinal cord.

Figure 1 also illustrates the degenerating axons which descend ipsilaterally within the lateral brainstem tegmentum and terminate in such laterally situated structures as the parabigeminal and dorsolateral pontine nuclei as well as in the dorsolateral part of the tegmental reticular nucleus (sections 65 and 57 ). Occasional degenerating fibers leave this ipsilateral descending pathway almost at right angles to course medially through the tegmentum (sections 65 and 57). As can be seen in section 57, some of these appear to terminate fairly close to the midline within the caudal subdivision of the pontine reticular nucleus.

To summarize, two major descending pathways leave the superior colliculus, a contralateral pathway which projects primarily to the tegmental reticular nucleus and paramedian pontine reticular formation and an ipsilateral pathway which distributes mainly to the pontine nuclei and to the lateral pontine reticular formation. In the results which follow, we will be concerned with the laminar origin of the crossed tectoreticular pathway.

Laminar source. In order to estimate the laminar source of the contralateral tectoreticular fibers, small injections of tritiated amino acids were confined to single grey lamina of the superior colliculus. In total, 13 restricted injections were made. Figure 2 summarizes the data derived from three representative cases.

Part $A$ illustrates the characteristic distribution of autoradiographic label which follows small injections restricted to the superficial grey lamina (stratum griseum superficiale). In none of the cases with superficial injections was label seen over the crossed tectoreticular pathway. However, prominent label was found over known targets of the superficial grey layer. For example, ascend-

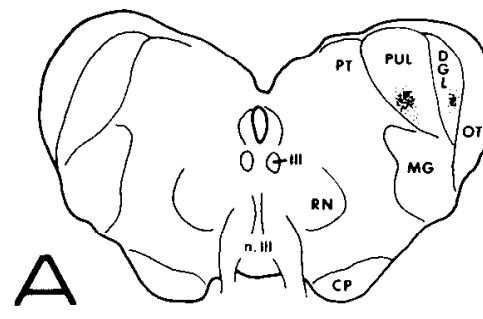

SUPERFICIAL
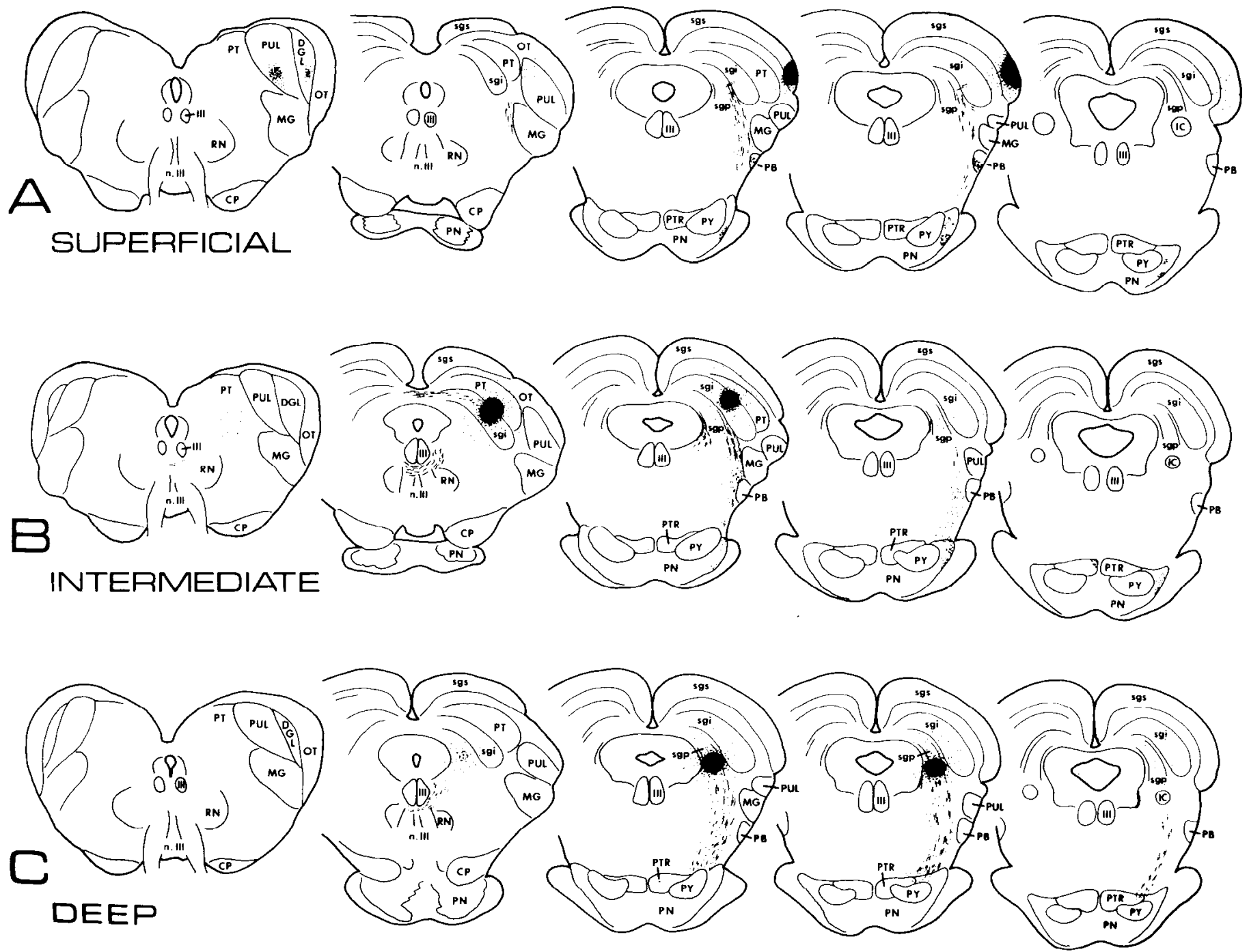

Figure 2. Three cases with injections of tritiated amino acids restricted to particular layers of the superior colliculus. In case $A$, the injection is restricted to the layers above the stratum opticum; in case $B$, the injection is in the intermediate layer with some spread into the pretectum. In case $C$, the injection is in the deep grey layer. Labeled fibers are indicated by lines and labeled terminals are represented by dots. The resulting distributions of anterogradely transported label are described in the text. Scale, $1 \mathrm{~mm}$. 
ing projections to the dorsal lateral geniculate nucleus and the pulvinar are labeled. In addition, labeled uncrossed descending fibers could be traced to the parabigeminal nucleus. These positive results are relevant since they demonstrate that the injection was sufficiently large to label known targets of the superficial layers (Harting et al., 1973; Benevento and Fallon, 1975; Robson and Hall, 1976, 1977; Holcombe and Hall, 1978, 1981a; Albano et al., 1979; Kawamura et al., 1980).

Part $B$ of Figure 2 illustrates a case in which the injection site is focused in the intermediate grey lamina (stratum griseum intermediale) of the superior colliculus with some spread into the adjacent pretectal nuclei. This injection apparently avoided the superficial grey lamina since no label could be detected over the dorsal and ventral lateral geniculate nuclei, the pulvinar, or the parabigeminal nucleus. Except for the absence of label over the parabigeminal nucleus and the presence of dense label over the midbrain tegmentum, the distribution of label over descending ipsilateral pathways in this case is similar to that following more superficial injections. Thus, both superficial and intermediate injections result in prominent label over ipsilateral tectofugal fibers. However, in contrast to more superficial injections, injections restricted to the intermediate grey lamina always produce conspicuous labeling of the crossed tectoreticular fibers. In Figure $2 B$, these labeled fibers can be traced through the deep grey lamina before they emanate ventrally from the superior colliculus. Their decussation also is labeled distinctly. Due to the small size of the injection and the rostrocaudal orientation of this fiber bundle, labeling over the caudal continuation of the contralateral tectoreticular pathway throughout the brainstem is difficult to detect. However, terminal label was clearly evident over the pontine tegmental reticular nucleus medially.

Part $C$ of Figure 2 illustrates the typical pattern of label resulting from injections of amino acids which are centered in the deep grey lamina (stratum griseum profundum) of the superior colliculus. In this case, the ipsilateral tectoreticular fibers are labeled prominently. However, no label is present over known targets of the superficial layers, such as the visually related thalamic structures and the parabigeminal nucleus. Most importantly, in contrast to the cases with injections in the intermediate grey lamina, only very light label is present over the contralateral descending pathway after injections restricted to the deep cellular lamina. As a general rule, when label is detected over the crossed tectoreticular pathway following injections of the deep grey lamina, only a few fibers can be traced crossing the midline and the presence of label over the contralateral paramedian tegmentum is ambiguous.

Figure 3 is a diagrammatic representation of three levels of a cross-section through the superior colliculus and summarizes all of the small amino acid injections made in this series of experiments. Each open circle represents an injection site which resulted in no detect. able label over the crossed tectoreticular pathway. Of seven such injection sites, six involved primarily the superficial grey lamina and one was centered in the deepest fiber lamina but included the deep half of the decp grey lamina. The four hatched circles represent injections which resulted in only very sparse label over the crossed tectoreticular pathway. Two of these injection sites were focused at the junction between the superficial grey lamina and the optic fibrous lamina (stratum opticum) but encroached slightly upon the intermediate grey lamina laterally. The two remaining injections which resulted in only minimal label over the crossed tectoreticular pathway were restricted to the deep grey lamina. In contrast, the two filled circles represent injection sites which resulted in prominent label over the crossed tectoreticular pathway. Both of these cases had injection sites which were restricted to or centered in the intermediate grey lamina.

To summarize, the contralateral tectoreticular pathway is not labeled following amino acid injections of the superficial grey lamina unless some of the intermediatc grey lamina is also included in the injection site. Likewise, the crossed tectoreticular bundle is labeled only sparsely following injections of the deep grey lamina. However, following injections of the intermediate grey lamina, the label over the contralateral tectoreticular pathway is prominent.

Cells of origin. In order to provide a more quantitative estimate of the cells of origin for the crossed tectoreticular pathway, HRP was injected into various locations along the course of this pathway. Figure 4 illustrates three cases in which HRP was injected into the area of

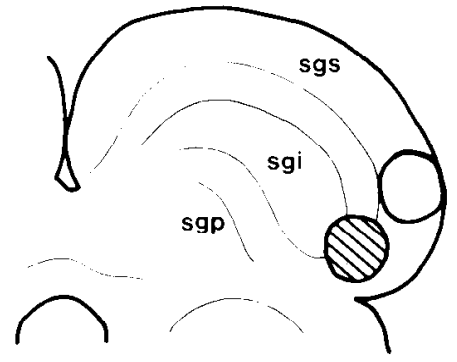

CAUDAL
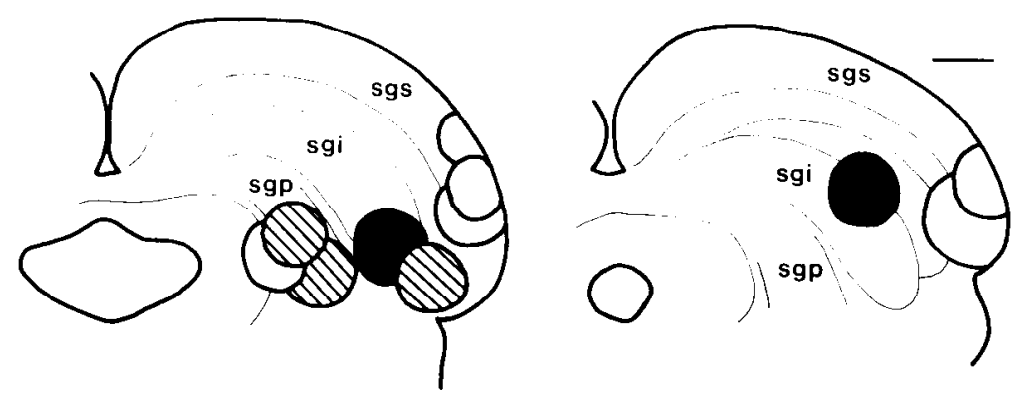

ROSTRAL

Figure 3. Summary of all restricted injections of tritiated amino acids. Fach filled circle represents an injection site which resulted in label over the crossed tectoreticular pathway. Each hatched circle represents an injection site which resulted in sparse label over the crossed tectoreticular pathway. Each open circle represents an injection site which resulted in no label over the crossed tectoreticular pathway. Scale, $1 \mathrm{~mm}$. 


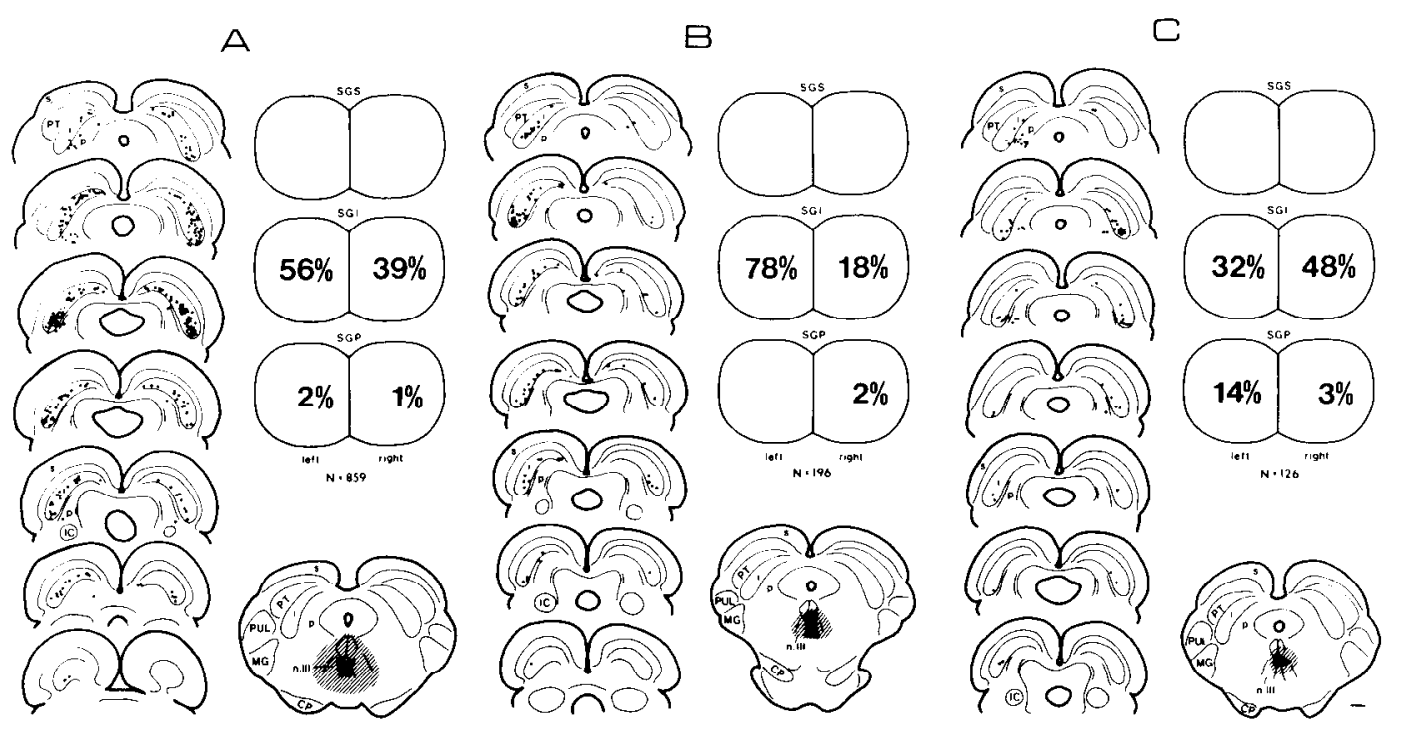

Figure 4. Laminar distribution of retrogradely labeled cells within the superior colliculus following injections of HRP in the decussation of the predorsal bundle. The injection sites are indicated to the lower right of the series of transverse sections through the superior colliculus. The most densely stained area of the injection site is indicated by black and the maximum extent of detectable spread is shown by hatching. The columns of transverse sections through the superior colliculus proceed from rostral at the top to caudal at the bottom. Each dot represents a labeled cell. At the upper right are dorsal reconstructions of these grey layers and the corresponding percentages of labeled cells located within each lamina. The total number of labeled neurons counted is indicated by $N$. Scale, $1 \mathrm{~mm}$.

decussating tectoreticular fibers. The most striking feature shared by these three cases is that the overwhelming majority $(95 \%, 96 \%$, and $80 \%)$ of the cells filled with reaction product are located in the intermediate grey lamina. The only notable exception is the small group of labeled cells in the rostral part of stratum griseum profundum in cases $A$ and $C$. Most of the remaining filled cells are scattered within either the intermediate or deep fibrous lamina. No labeled cells were found in the superficial lamina. The filled cells appear to be distributed throughout the rostrocaudal as well as the mediolateral dimensions of the superior colliculus. The results from these three cases support the conclusion that the crossed tectoreticular pathway originates chiefly from the intermediate grey lamina but also, to a much lesser degree, from the deep grey layer.

In order to estimate more precisely the relative contributions of the intermediate and deep laminae to the crossed tectoreticular pathway, very small injections of HRP were made in the paramedian tegmentum near the pontomedullary junction (refer to section 49 of Fig. 1). Two such cases are illustrated in Figure 5. It should be noted that the injection sites were so small that only a few cells were filled in the superior colliculus in each case ( 16 and 37 cells, respectively). However, despite the small number of filled cells, the results are consistent and confirm the results of the anterograde experiments. In both cases, the great majority ( $88 \%$ ) of labeled cells are located in the contralateral intermediate grey lamina and the remainder are located contralaterally within the deep lamina. No filled cells are located in the contralateral superficial lamina or in any laminae of the ipsilateral superior colliculus. Thus, even with tiny injections caudal

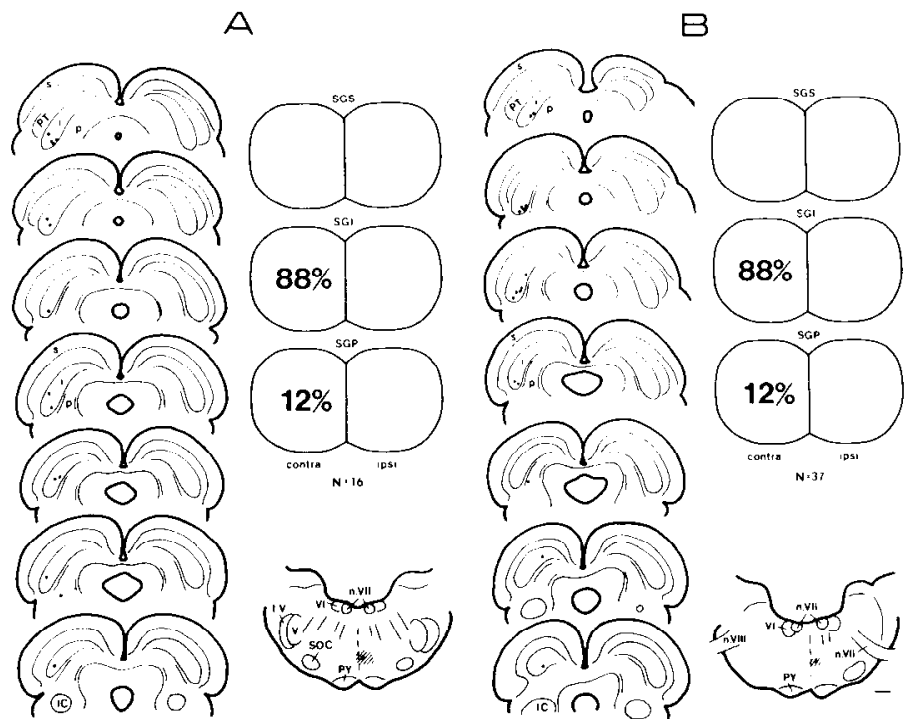

Figure 5. Laminar distribution of retrogradely labeled cells in the superior colliculus following injections of HRP within the paramedian reticular formation. See the legend to Figure 4 for a description of the figure layout. Scale, $1 \mathrm{~mm}$.

to the decussation, the labeled cells are located primarily in the intermediate lamina. Only a few filled cells are present within the deep lamina.

This difference in the relative contribution of the intermediate and deep laminae to the crossed tectoreticular projection system is a consistent finding even following large injections of HRP. Figure 6 illustrates three such cases. The probable uptake zone of the injection site illustrated in Figure $6 A$ occupies a large tegmental area 

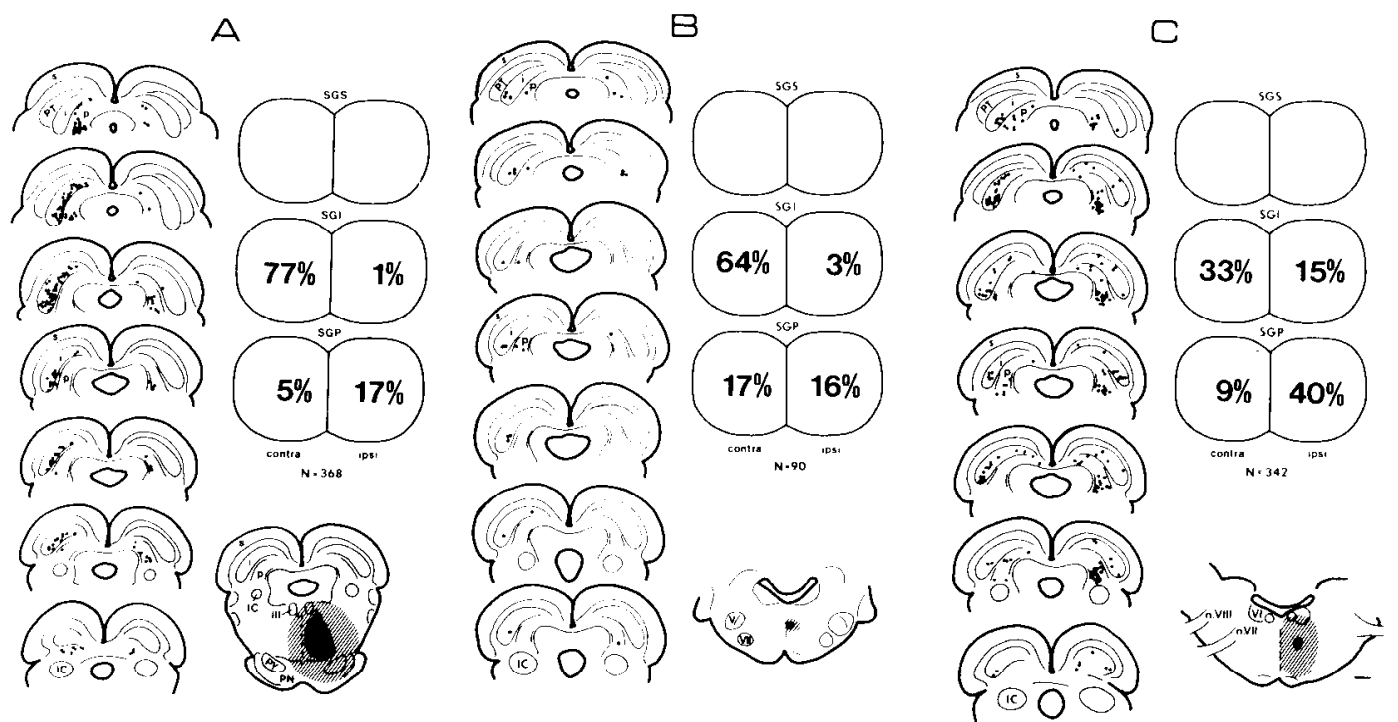

Figure 6. Laminar distribution of retrogradely labeled cells within the superior colliculus following injections of HRP in the reticular formation. Case $A$ is an example of an extensive injection; case $B$ has a small medial injection and case $C$ shows an injection which is situated more laterally. See the legend to Figure 4 for a description of the figure layout. Scale, $1 \mathrm{~mm}$.

extending as far dorsal as the oculomotor nucleus and as far ventral as the pontine nuclei. Even following such an extensive injection, the majority of filled cells in the contralateral superior colliculus (77\%) are located in the intermediate grey lamina with a relatively small contribution from the rostral part of stratum griseum profundum. However, in contrast to the cases depicted in Figure 5 , this injection also resulted in filled cells in the ipsilateral superior colliculus, most of which are located within the deep grey lamina. We attribute the filling of these ipsilateral cells to the extension of the injection site laterally (Henkel and Edwards, 1978; Holcombe and Hall, 1981b).

The injection site in Figure $6 B$ is smaller than the one just described but larger than those depicted in Figure 5 . Once again, the relative contributions of the contralateral laminae are consistent with the findings already described: that is, the majority of filled cells are located within the intermediate grey lamina. The injection site in this case extends further laterally than those of Figure 5 and, as would be expected, filled cells are found in the deep grey lamina on the ipsilateral side.

Finally, Figure $6 C$ illustrates a medium size injection which is located further from the midline than those illustrated in Figure $6, A$ and $B$. One result of this lateral placement is an increase in the relative number of labeled cells found ipsilaterally. As in previous cases, the majority of these ipsilateral filled cells are located in the deep cellular lamina. The injection site clearly still involves the terminal field of the crossed tectoreticular projection system since many filled cells are located in the contralateral superior colliculus. As in all cases described so far, the majority of these contralateral cells are located in the intermediate grey lamina.

In summary, whether HRP injections are confined strictly to the paramedian reticular formation or extend laterally from this area, the results indicate that the majority of crossed tectoreticular fibers originate from the intermediate grey lamina. When the injections extend laterally, they encroach upon the tegmental area which receives uncrossed tectoreticular projections and, as a result, labeled cells are located predominantly within the deep grey lamina on the same side as the injection.

\section{Discussion}

Course and distribution of the crossed tectoreticular pathway. As in other species, the superior colliculus of the grey squirrel is the source of a prominent pathway which projects to the contralateral paramedian reticular formation. Most of the axons which form this pathway terminate in the pons either rostral to or at the level of the abducens nucleus and nerve. Since a very similar course and distribution has been found in a wide variety of species, including non-mammalian vertebrates (Ebbesson, 1970; Harting et al., 1973; Kawamura et al., 1974; Foster and Hall, 1975; Harting, 1977), it seems reasonable to infer that this pathway has been a constant feature of tectal organization since the earliest stages of vertebrate evolution.

The terminal distribution of the crossed tectoreticular fibers supports the view that tectal influences on brainstem and spinal motor neurons concerned with head and eye movements are mediated chiefly through the reticular formation (Anderson et al., 1971). Although a few tectofugal fibers may contact motor neurons directly in some species (Harting, 1977; Edwards and Henkel, 1978), no evidence for such projections was found either in the present study of the grey squirrel or in similar studies of the rabbit (Tarlov and Moore, 1966), opossum (Martin, 1969), or tree shrew (Harting et al., 1973). Furthermore, even when present, these projections are sparse, as are direct projections from the tectum to the spinal cord. In comparison, prominent pathways can be traced from the paramedian pontine reticular formation to the abducens nucleus as well as to adjacent regions, such as nucleus 
prepositus hypoglossi and the dorsomedial medullary reticular formation caudal to the abducens nucleus (Graybiel and Hartwieg, 1974; Buttner-Ennever and Henn, 1976). All of these regions have been implicated in the control of eye movements and project to extraocular motor nuclei (Graybiel and Hartwieg, 1974; Buttner-Ennever and Henn, 1976; Grantyn et al., 1977). Similarly, a group of neurons which lies within the terminal distribution of the predorsal bundle just rostral to the abducens nucleus projects to the spinal cord and may provide an analogous pathway for controlling orienting movements of the head (Peterson et al., 1974; Kuypers and Maisky, 1975; Grantyn et al., 1979).

Although the distribution of crossed fibers to the paramedian reticular formation is very constant among the species which have been studied, variations have been described in the crossed projections to other lower brainstem targets. More specifically, we did not find evidence for a pathway to the inferior olive of the grey squirrel following lesions restricted to the superior colliculus, whereas tecto-olivary pathways have been described in other species including at least two primates (Frankfurter et al., 1976; Harting, 1977), the tree shrew, (Harting et al., 1973), and the cat (Graham, 1977). Also, we did not observe the heavy projection from the tectum to the nucleus reticularis gigantocellularis of the medulla which has been described in the cat (Kawamura et al., 1974). At present, it is uncertain whether these differences reflect the techniques used or true species variations. However, there is sufficient evidence to suggest that the size of the tecto-olivary projection does vary among species. For example, this pathway was seen only after very large tectal lesions in the tree shrew (Harting et al., 1973) and apparently is only demonstrable with autoradiographic anterograde techniques in the cat (Kawamura et al., 1974; Frankfurter et al., 1976; Graham, 1977). Similarly, only a few neurons in the deep tectal layers were labeled retrogradely after large olivary injections of HRP in the opossum (Henkel et al., 1975). In the latter study, many more retrogradely labeled cells were found in the underlying mesencephalic reticular formation and in the periaqueductal grey. In contrast, the tecto-olivary pathway appears to be quite prominent in primates and apparently arises within the deep layers of the superior colliculus (Frankfurter et al., 1976; Harting, 1977).

Laminar origin of the crossed tectoreticular pathway. The most important finding of the present experiments is that the cells of origin for the crossed tectoreticular pathway are restricted almost entirely to the intermediate grey layer, stratum griseum intermediale. This result is consistent with those of previous studies using anterograde techniques (Harting et al., 1973; Graham, 1977; Harting, 1977), which indicated that this pathway arises in the deep half of the colliculus, but extends them by demonstrating that the majority of fibers arise in the intermediate rather than in the deep grey layer. Also consistent with this conclusion is the finding that cells residing in the intermediate layer project contralaterally to extraoculomotor regions in the cat (Edwards and Henkel, 1978).

At least two previous studies presented results suggesting that the deep layer, stratum griseum profundum, is the major source of the predorsal bundle (Graham, 1977; Raczkowski and Diamond, 1978). Graham (1977), relying on the anterograde transport of tritiated amino acids, suggested that the intermediate grey layer in the cat is primarily a zone of interneurons and is the origin of few, if any, descending projections. However, in a more recent study of the same species which employed retrograde transport of HRP following injections in the paramedian reticular formation, Kawamura and Hashikawa (1978) reported that the majority of labeled cells were located in the intermediate grey layer. This discrepancy may be due to the different techniques since the small injections of tritiated amino acids in the intermediate grey layer made by Graham may have been insufficient to produce detectable levels of label over the descending pathways. Similarly, in our experiments on the grey squirrel, this pathway was difficult to trace in transverse sections after small injections because the labeled fibers are few in number, scattered, and oriented perpendicular to the plane of section. However, as in the cat, retrograde transport experiments provided unequivocal evidence that the intermediate grey layer in the squirrel is the main source of the predorsal bundle.

Results of a recent study of the galago (Raczkowski and Diamond, 1978), which suggested that this pathway arises from the deep grey layer, are more difficult to reconcile with those from the cat and squirrel since they too are based upon retrograde transport. However, one possible source of this apparent discrepancy might be the cytoarchitectonic definition of the intermediate and deep layers in species in which these layers are difficult to distinguish. Alternatively, since some neurons in the deep grey layer do contribute to the predorsal bundle in both the squirrel (see Figs. 4 and 6) and cat (Kawamura and Hashikawa, 1978; Edwards, 1980), it seems plausible that the corresponding cells in the galago were labeled preferentially, either because of species differences in the relative contribution of each layer or because of differential labeling due to an unknown topographical organization of the fibers in the predorsal bundle.

Organization of the superior colliculus. Traditionally, the superior colliculus has been subdivided into two main parts, a superficial and a deep zone. The superficial zone, which includes the grey layer above the stratum opticum, receives input mainly from the retina and occipital lobe and projects primarily to visually related nuclei of the thalamus and midbrain. The term "deep zone" usually is used to refer collectively to the intermediate and deep grey layers. These layers receive input from several sensory systems and are considered to be the major source of the descending pathways to lower brainstem motor areas.

One important implication of our studies in the grey squirrel is that a fundamental distinction can be made between the intermediate and deep grey layers within the deep zone. In the experiments described in this paper, at least $80 \%$ of the retrogradely labeled cells were found consistently in the contralateral intermediate grey layer following injections in the paramedian reticular formation. In contrast, in a previous study, we found that, following injections in the lateral reticular formation, over $80 \%$ of the labeled cells were located ipsilaterally 
and in the deep rather than in the intermediate grey layer (Holcombe and Hall, 1981b). We do not know the significance of these differences, but they do suggest that a functional distinction can be made between the intermediate and deep grey layers of the superior colliculus which is related to the distinctions others have made between the magnocellular medial and parvocellular lateral parts of the brainstem reticular formation (Brodal, 1957; Nauta and Kuypers, 1958). Distinctions between cells in the intermediate and deep grey layers also have been made by physiologists on the basis of differences in response lead time before the onset of saccades and in the sizes of the visual receptive fields (Mohler and Wurtz, 1976).

This distinction between the intermediate and deep layers on the basis of differences in efferent pathways raises the question of the laminar distribution of inputs to the deep zone. Although numerous sources of inputs, including the overlying superficial layers (Sprague, 1975), brainstem sensory relay nuclei (Edwards et al., 1979), and various cortical areas (Sprague, 1975; Goldman and Nauta, 1976; Kunzle and Akert, 1977; Wise and Jones, 1977), have been proposed in previous studies, relatively little information concerning the laminar distribution of each within the deep layers is available. From the present results, it is evident that a more precise delineation of these terminal zones will be necessary before we can characterize the inputs to the cells of origin for the descending tectofugal pathways involved in the control of gaze.

\section{References}

Albano, J. E., T. T. Norton, and W. C. Hall (1979) Laminar origin of projections from the superficial layers of the superior colliculus in the tree shrew, Tupaia glis. Brain Res. 173: 111 .

Anderson, M. E., M. Yoshida, and V. J. Wilson (1971) Influence of superior colliculus on cat neck motoneurons. J. Neurophysiol. 34: 898-907.

Benevento, L. A., and J. H. Fallon (1975) 'The ascending projections of the superior colliculus in the rhesus monkey (Macaca mulatta). J. Comp. Neurol. 160: 339-362

Brodal, A. (1957) The Reticular Formation of the Brain Stem: Anatomical Aspects and Functional Correlations, The Henderson Trust Lecture. Oliver \& Boyd, Edinburgh.

Buttner-Ennever, J. A., and V. Henn (1976) An autoradiographic study of the pathways from the pontine reticular formation involved in horizontal eye movements. Brain Res. 108: 155-164.

Casagrande, V. A., and I. T. Diamond (1974) Ablation study of the superior colliculus in the tree shrew (Tupaia glis). J. Comp. Neurol. 156: 207-238.

Ebbesson, S. O. E. (1970) On the organization of central visual pathways in vertebrates. Brain Behav. Evol. 3: 178-194.

Edwards, S. B. (1980) The deep layers of the superior colliculus: Their reticular characteristics and structural organization. In The Reticular Formation Revisited, J. A. Hobson and M. A. Brazier, eds., pp. 193-209, Raven Press, New York.

Edwards, S. B., and C. K. Henkel (1978) Superior colliculus connections with the extraocular motor nuclei in the cat. J. Comp. Neurol. 179: 451-467.

Edwards, S. B., C. L. Ginsburg, C. K. Henkel, and B. E. Stein (1979) Sources of subcortical projections to the superior colliculus in the cat. J. Comp. Neurol. 184: 309-330.

Fink, R. P., and L. Heimer (1967) Two methods for selective silver impregnation of degenerating axons and their synaptic ends in the central nervous system. Brain Res. 4: 369-374.

Foster, R. E., and W. C. Hall (1975) The connections and laminar organization of the optic tectum in a reptile (Iguana iguana). J. Comp. Neurol. 163: 397-426.

Frankfurter, A., J. T. Weber, G. J. Royce, N. L. Strominger, and J. K. Harting (1976) An autoradiographic analysis of the tecto-olivary projection in primates. Brain Res. 118: 245-257.

Goldman, P. S., and W. J. H. Nauta (1976) Autoradiographic demonstration of a projection from prefrontal association cortex to the superior colliculus in the rhesus monkey. Brain Res. 116: 145-149.

Graham, J. (1977) An autoradiographic study of the efferent connections of the superior colliculus in the cat. J. Comp. Neurol. 173: 629-654.

Grantyn, A., and A. Berthoz (1977) Synaptic actions of the superior colliculus on medial rectus motoneurons in the cat. Neuroscience 2: 945-951.

Grantyn, A., and R. Grantyn (1976) Synaptic actions of tectofugal pathways on abducens motoneurons in the cat. Brain Res. 105: 269-285.

Grantyn, A., R. Grantyn, K. P. Robine, and A. Berthoz (1979) Electroanatomy of tectal efferent connections related to eye movements in the horizontal plane. Exp. Brain Res. 37: 149172

Graybiel, A. M., and E. A. Hartwieg (1974) Some afferent connections of the oculomotor complex in the cat: An experimental study with tracer techniques. Brain Res. 81: 543-551.

Harris, L. R. (1980) The superior colliculus and movements of the head and eyes in cats. J. Physiol. (Lond.) 300: 367-391.

Harting, J. K. (1977) Descending pathways from the superior colliculus: An autoradiographic analysis in the rhesus monkey (Macaca mulatta). J. Comp. Neurol. 173: 583-612.

Harting, J. K., W. C. Hall, I. T. Diamond, and G. F. Martin (1973) Anterograde degeneration study of the superior colliculus in Tupaia glis: Evidence for a subdivision between superficial and deep layers. J. Comp. Neurol. 148: 361-386.

Hendrickson, A. L., L. Moe, and B. Noble (1972) Staining for autoradiography of the central nervous system. Stain Technol. 47: 283-290.

Henkel, C. K., and S. B. Edwards (1978) The superior colliculus control of pinna movements in the cat: Possible anatomical connections. J. Comp. Neurol. 182: 763-776.

Henkel, C. K., M. Linauts, and G. F. Martin (1975) The origin of the annulo-olivary tract with notes on other mesencephaloolivary pathways. A study by the horseradish peroxidase method. Brain Res. 100: 145-150.

Holcombe, V., and W. C. Hall (1978) Laminar origin of two descending pathways from the superior colliculus in the grey squirrel. Soc. Neurosci. Abstr. 4: 632.

Holcombe, V., and W. C. Hall (1981a) Course and laminar origin of the tectoparabigeminal pathway. Brain Res. 211: 405-411.

Holcombe, V., and W. C. Hall (1981b) The laminar origin of ipsilateral tectopontine pathway. Neuroscience 6: 255-260.

Kawamura, K., and T. Hashikawa (1978) Cell bodies of origin of reticular projections from the superior colliculus in the cat: An experimental study with the use of horseradish peroxidase as a tracer. J. Comp. Neurol. 182: 1-16.

Kawamura, K., A. Brodal, and G. Hoddevik (1974) The projection of the superior colliculus onto the reticular formation of the brain stem. An experimental anatomical study in the cat. Exp. Brain Res. 19: 1-19.

Kawamura, S., N. Fukushima, S. Hattori, and M. Kudo (1980) Laminar segregation of cells of origin of ascending projections from the superficial layers of the superior colliculus in the cat. Brain Res. 184: 486-490.

Kunzle, II., and K. Akert (1977) Efferent connections of cortical 
area 8 (frontal eye field) in Macaca fascicularis. A reinvestigation using the autoradiographic technique. J. Comp. Neurol. 173: 147-164.

Kuypers, H. B. J. M., and V. A. Maisky (1975) Retrograde axonal transport of horseradish peroxidase from spinal cord to brain stem cell groups in the cat. Neurosci. Lett. 1: 9-14.

Martin, G. F. (1969) Efferent tectal pathways of the opossum (Didelphis virginiana). J. Comp. Neurol. 135: 209-224.

Mohler, C. W., and R. H. Wurtz (1976) Organization of monkey superior colliculus: Intermediate layer cells discharging before eye movements. J. Neurophysiol. 39: 722-774.

Nauta, W. J. H., and G. J. M. Kuypers (1958) Some ascending pathways in the brain stem reticular formation. In Reticular Formation of the Brain, H. H. Jasper, ed., pp. 3-30, Little, Brown and Co., Boston.

Peterson, B. W., M. E. Anderson, and M. Filion (1974) Responses of pontomedullary reticular neurons to cortical, tectal and cutaneous stimuli. Exp. Brain Res. 21: 19-44.

Raczkowski, D., and I. T. Diamond (1978) Cells of origin of several efferent pathways from the superior colliculus in Galago senegalensis. Brain Res. 146: 351-357.

Raczkowski, D., V. A. Casagrande, and I. T. Diamond (1976) Visual neglect in the tree shrew after interruption of the descending projections of the deep superior colliculus. Exp. Neurol. 50: 14-29.

Raybourn, M. S., and E. L. Keller (1977) Colliculoreticular organization in primate oculomotor system. J. Neurophysiol. 40: 861-878.

Robinson, D. A. (1972) Eye movements evoked by collicular stimulation in the alert monkey. Vision Res. 12: 1795-1808.

Robson, J. A., and W. C. Hall (1976) Projections from the superior colliculus to the dorsal lateral geniculate nucleus of the grey squirrel (Sciurus carolinensis). Brain Res. 113: 379385.

Robson, J. A., and W. C. Hall (1977) Organization of the pulvinar in the grey squirrel (Sciurus carolinensis). I. Cytoarchitecture and connections. J. Comp. Neurol. 173: 355388 .

Schiller, P. H., and F. Koerner (1971) Discharge characteristics of single units in superior colliculus of the alert rhesus monkey. J. Neurophysiol. 34: 920-936.

Schiller, P. H., and M. Stryker (1972) Single-unit recording and stimulation in superior colliculus of the alert rhesus monkey. J. Neurophysiol. 35: 915-924.

Schneider, G. E. (1967) Contrasting visuomotor functions of tectum and cortex in the golden hamster. Psychol. Forsch. 31: $52-62$.

Schneider, G. E. (1969) Two visual systems. Science 163: 895902.

Sprague, J. M. V. (1975) Mammalian tectum: Intrinsic organization, afferent inputs and integrative mechanisms. Neurosci. Res. Program Bull. 13: 204-213.

Stryker, M. P., and P. H. Schiller (1975) Eye and head movements evoked by electrical stimulation of monkey superior colliculus. Exp. Brain Res. 23: 103-112.

Tarlov, E. C., and R. Y. Moore (1966) The tecto-thalamic connections in the brain of the rabbit. J. Comp. Neurol. 126: 403-421.

Wise, S. P., and E. G. Jones (1977) Somatotopic and columnar organization in the corticotectal projection of the rat somatic sensory cortex. Brain Res. 133: 223-235.

Wurtz, R. H., and M. E. Goldberg (1972) Activity of superior colliculus in behaving monkey. III. Cells discharging before eye movements. J. Neurophysiol. 35: 575-586. 